NBER WORKING PAPFR SERIFS

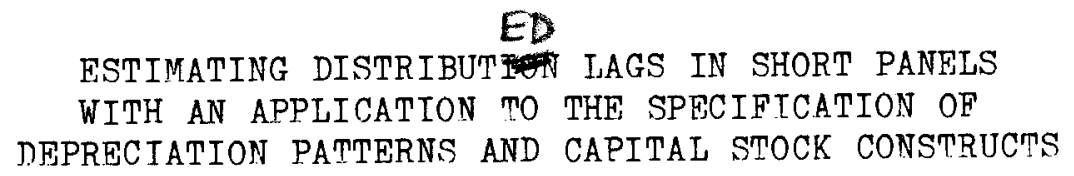

Ariel Pakes

Zvi Griliches

Working Paper No. $\underline{933}$

NATIONAL BUREAU OF ECONOMIC RESFARCH 1050 Massachusetts Avenue Cambridge MA 02138

July 1982

The research reported here is part of the NBER's research program in Productivity. Any opinions expressed are those of the authors and not those of the National Bureau of Economic Research. 


\section{Estimating Distribution Lags in Short Panels with an Application to the Specification of Depreciation Patterns and Capita1 Stock Constructs}

\section{ABSTRACT}

In this paper, we investigate the problem of estimating distributed lags in short panels. Estimates of the parameter of distributed lag relationships based on single time-series of observations have been usually rather imprecise. The promise of panel data is in the $\mathrm{N}$ repetitions of the time-series that it contains which should allow one to estimate the identified lag parameters with greater precision. On the other hand, panels tend to track their observations only over a relatively short time interval. Thus, some assumptions will have to be made on the contribution of the unobserved presample $x$ 's to the current values of $y$ before any lag parameters can be identified from such data. In this paper we suggest two such assumptions; both of which are, at least in part, testable, and outline appropriate estimation techniques. The first places reasonable restrictions on the relationship between the presample and insample $x$ 's, while the second imposes conventional functional form constraints on the lag coefficients. The paper concludes with an example which investigates empirically how to construct a "capital stock" for profit or rate of return regressions.

Ariel Pakes

National Bureau of Economic Research 1050 Massachusetts Avenue Cambridge, MA 02138

(617) $868-3921$

Zvi Griliches National Bureau of Economic Research 1050 Massachusetts Avenue Cambridge, MA 02138

(617) 495-2181 
ESTIMATING DISTRIBUTED LAGS IN SHORT PANELS WITH AN APPLICATION TO THE SPECIFICATION OF DEPRECIATION PATTERNS AND

CAPITAL STOCK CONSTRUCTS

\section{INTRODUCTION}

The problem we deal with in this paper arises, as is often the case in econometrics, because we do not have all the data that we would like to have. We often expect that changes in our independent variables have relatively long lasting effects, but have only fairly short time series to uncover them. Consider, for example, the simple model

$$
y_{t}=w_{0} x_{t}+w_{1} x_{t-1}+\cdots+w_{m} x_{t-m}+u_{t}
$$

where we have suppressed the constant term for simplicity of exposition. Here $\mathrm{m}+1$ is the total length of the lag structure and the problem we wish to consider arises when $m$ is large relative to $T$, or to $\mathrm{T}+\theta$, where $\mathrm{T}$ is the total number of observations on $\mathrm{y}$, and $\theta$ is the available number of lagged observations on $x \cdot A$ typical example might be $T=20, m=6$, and $\theta=2$. In this case, one would have to give up 4 observations on $y$ to get the whole lag "in", and estimate 8 parameters (including the constant term) on the basis of 16 observations. The resulting estimates are unlikely to be very precise. One way of gaining precision is to impose additional structure on the $w^{\prime} s$, choosing the form of the lag a priori -- polynomial, geometric, etc. Alternatively, one may try to increase the sample in some other dimension, adding more individuals, states or commodities. The sample becomes then a time-series panel with $\mathrm{N} \times \mathrm{T}$ observations. 
A significant number of panel data sets are now available. Though $\mathbb{N}$ in these data sets is often quite large, it is not yet clear whether they will yield "better" estimates of distributed lag coefficients. Part of the problem lies in the fact that panel data sets usually track their observations only over a rather short time interval. Thus, if in the example introduced above $T+\theta$ were less than seven while $m>7$, we could not estimate any of the coefficients consistently (as $\mathbb{N}$ grows large) without bringing in additional information. The problem is, of course, that in short panels the contribution of the unobserved presample $x^{\prime} s$ to the current values of $\mathrm{y}$ (or the truncation remainders) are likely to be particularly important. Since the value of the truncation remainder is determined by the lag coefficients and the presample $x^{\prime} s$ one has to constrain either the lag structure, or the stochastic process generating these $x^{\prime} s$, in order to identify any of the coefficients of interest. The first alternative has been discussed in the context of a single time series by Klein (1958), Dhrymes (1971), Maddala and Rao (1971), Pesaren (1973), and others. In Section III, we discuss how their solutions can be adapted to the panel data situation. Because there are usually many more degrees of freedom available in panel data, we concentrate on solutions which do not restrict the lag coefficients a priori. Section II considers, therefore, the problem of estimating a sequence of unrestricted lag coefficients when one is willing to impose some structure on the stochastic processes generating the $x^{\prime} s$. The models we deal with allow for the possibility of individualspecific time-invariant unobservable factors which impact both on $y$ given past $x^{\prime} s$ and on the distribution of the $x^{\prime} s$ themselves (they 
allow for heterogeneity and correlated effects). We consider explicitly the identification of lag coefficients when the regression function of recent $x^{\prime} s$ on all past values of $x$ and an individual specific constant term depends only on this constant term and a small number of the immediately preceeding $x^{\prime} s$. Examples of stochastic processes which statisfy such assumptions are mixture processes consisting of individual-specific components plus an integrated autoregressive deviate. The parameters defining this process may differ from year to year (they need not be homogeneous over time). It is shown that given this assumption one can identify the leading lag coefficients without making any assumptions on the shape of the rest of the lag distribution and test for alternative simplifications (for, e.g., that there is no individual-specific component, or that the lag coefficients, at least after a few free lags, have an autoregressive structure). These assumptions do not allow, however, for the identification of the tail of the lag structure (at least not without further restrictions). One cannot provide answers to questions involving the entire sequence of the lag coefficients (such as their sum) without imposing constraints on the lag structure itself (Sims, 1974). On the other hand, the early w's are often of significant interest in themselves, especially since unconstrained estimates of their values may provide an empirical basis for restricting the tail of the lag structure in further work. This brings us then to Section III where we discuss the estimation of distributed lag coefficients from panel data when one is willing to restrict the shape of the lag structure a priori. 
An advantage of using the approach discussed here is that, in general, it can be checked against the observed behavior of the data. The tests we consider consist of a set of constraints on the second order population moments of the vector $\underset{\sim_{i}}{r}=\left(\underset{\sim_{i}}{y^{\prime}}, \underset{\sim_{i}}{x^{\prime}}\right)$; where $y_{\sim_{i}}$ is the $T$ element vector of observations on $y$ for individual $i$, and $\underset{\sim}{x}$ is the $T+\theta$ vector of observations on $x$. In most cases these constraints are restrictions on the matrix of coefficients obtained from the regression of each of the $T y_{i t}$ 's on all $(T+\theta)$ of the $x_{i t}$ 's. In each of our models this natrix, labelled II by Chamberlain (1978), consists of a combination of the lag coefficients of interest, and of the regression functions of the unobserved components (the truncation remainders, and the individualspecific sources of heterogeneity), on $\underset{\sim_{i}}{x_{1}}$.

We conclude with an empirical example. It consists of regressing the operating profits of firms on a distributed lag of their past investment expenditures. The example, therefore, investigates empirically how to construct a "capital stock" for profit or rate of return regressions. We find that the lag coefficients rise monotonically over the first three periods and then remains fairly constant over the next four or five. This differs significantly from lag structures based on straight line or declining balance depreciation schemes (in both of these the lag coefficients decline monotonically in $\tau$ ). When we compare the fit of the equations estimated from our models to the fit of a system of regressions functions based on the usual capital stock constructs (net or gross), our equations do quite a bit better. On the other hand, when we compare the restricted $I$ matrix 
implied by our model to an unrestricted II matrix, we find that we can, using traditional testing procedures, reject the null hypothesis embodied in our assumptions. It is doubtful whether the relationship between profits and past investments is truly stable over different firms and time periods.

II. DISTRIBUTED LAGS FROM PANEL DATA WITH PRIOR STRUCTURE ON THE $X$-PROCESS

The distributed lag model which we assume is given by $\mathrm{Al}$.

Al: $\quad y_{i t}=\alpha_{i}+\sum_{\tau=0}^{\infty} w_{\tau} x_{i t-\tau}+u_{i t}$

where, if $x_{i}^{\prime}=\left[x_{i}-\theta, \ldots, x_{i T}\right]$ and $\stackrel{\sim}{u}_{i}^{\prime}=\left[u_{i t}, \ldots, u_{i T}\right]$, it is assumed that the $N$ observations on the vector $\left(x_{i}^{\prime},{\underset{i j}{u}}_{i} \alpha_{i}\right)$ are independent draws from a common distribution function in which $E^{*}\left[u_{i} \mid x_{i}, D_{i}\right]=0$, where $D_{i}$ denotes the indicator function which takes the value of one for individual $i$ and zero elsewhere, and $\Sigma_{x x^{\prime}}=E\left[\underset{\sim i}{x}, x_{i}^{\prime}\right]$ is positive definite. The sequence $\left\{_{\tau}\right\}_{\tau=0}$ is a set of unknown finite parameters.

Here (and in the discussion below) $E^{*}\left(z_{1} \mid z_{2}\right)$ is the operator which provides the minimum mean square error linear predictor (or the regression function) of $z_{1}$ given $z_{2}$. Some preliminary comments on Al are in order. For ease of exposition we have omitted time-specific constant terms from it and from all equations to be introduced below. Since we will be concerned with the limiting distribution of the parameter estimates in dimension $\mathrm{N}$ (holding $\mathrm{T}$ fixed), time-specific 
constant terms, if required, can be added without creating any additional estimation problems. Al does assume that the distributed lag coefficients do not differ either over time or between individuals (though random differences in these coefficients as defined for example by Swamy, 1970, are covered by Al). Note, however, that the model does allow for individual-specific constant terms (the $\alpha_{i}$ ). This permits one to deal explicitly with the problem of unobserved individual-specific components which are constant over time and affect both the conditional distribution of $\mathcal{X}_{i}$ given the $x$ sequence corresponding to individual $i$ and the marginal distribution of $x_{i}$. Given the $\alpha_{i}$, we are also assuming that $u_{i t}$ is not correlated with either the $x^{\prime} s$ observed prior to, or those observed after, period $t\left[i . e ., E^{*}\left(u_{i} \mid{\underset{\sim}{i}}_{i}, D_{i}\right)=0\right]$. Since the models presented here will, in general, be overidentified, one may, in any particular example, wish to relax the assumptions of constancy of the lag parameters and $E^{*}\left(u_{i} \mid x_{i}, D_{i}\right)=0$. These assumptions do, however, allow for a relatively straightforward exposition of our results concerning the estimation of lag structures in short panels.

It is convenient to rewrite $A l$ as

$$
y_{i t}=\alpha_{i}+\sum_{\tau=0}^{t+\theta} w_{\tau} x_{i t-\tau}+b_{i t}+u_{i t}
$$

where $b_{i t}=\sum_{\tau=\theta+1}^{\infty} w_{t+\tau} x_{i-\tau}$, for $i=1, \ldots, N$ and $t=1, \ldots, T$.

In (1), $b_{i t}$ is the truncation remainder (or the contribution of presample $x$ to the current value of $y$ ) for individual $i$ in 
period $t$. Let the matrix of coefficients obtained from the regression function of ${\underset{\sim}{i}}_{i}$ on $\underset{\sim}{x}$ be $I$, defined by $E^{*}\left(y_{\sim i} \mid{\underset{\sim i}{i}}_{i}\right)=\underset{\sim}{\operatorname{IIx}}$. We will say that a lag coefficient is identified if it can be calculated from the elements of $\Pi$ II Given AI, equation (1) makes it clear that each row of $I$ will contain a combination of the lag coefficients of interest and of the coefficients from the regression functions of the two unobserved components, $\alpha_{i}$ and $b_{i t}$, on ${\underset{\sim}{i}}_{i}$. Our problem is, therefore, to separate out the lag coefficients from the coefficients defining these two regression functions.

The distinction between $\alpha_{i}$ and $b_{i t}$ is that $\alpha_{i}$ is constant over time while $b_{\text {it }}$ is not. The estimation of models containing the $\alpha_{i}$ has been discussed extensively in the past (see Mundlak, 1978, and the literature cited there) and therefore the particular difficulties we shall encounter in identifying (at least some of) the lag coefficients from $\Pi$ in our models arise from the existence of the truncation remainders (the $b_{i t}$ ). Since $b_{i t}=\sum_{\tau=\theta+1}^{\infty} w_{t+\tau} x_{i-\tau}$, in order to identify any one of the lag coefficients one has to restrict either the sequence of lag coefficients (the $w_{\tau}$ ), or the relationship between presample $x^{\prime} s$ and the observed data. This section deals with the second alternative.

1. This will be shown to be equivalent to stating that a lag coefficient is identified if it can be calculated from the second order population moments of $\underset{\sim i}{r} \underset{i}{\prime}\left(\underset{\sim i}{x_{i}^{\prime}}, y^{\prime}\right)$. 


\section{II.1 The Identification Problem When There Is No Heterogeneity}

The phrase 'no heterogeneity' will be used to denote a situation in which it is appropriate to assume that

A2:

$$
\alpha_{i}=0, \text { for } i=1, \ldots, N
$$

Since the basic ideas underlying our results are easier to grasp when we maintain A2, and since the addition of the $\alpha_{i}$ does not change the identification status of the lag coefficients in the models discussed here, we begin with this special case.

Given both $A 1$ and $A 2$ the $I$ matrix (which, in this special case, we denote by $\pi^{\text {nh }}$ ) has the form

$$
I I^{n h}=W+B
$$

where

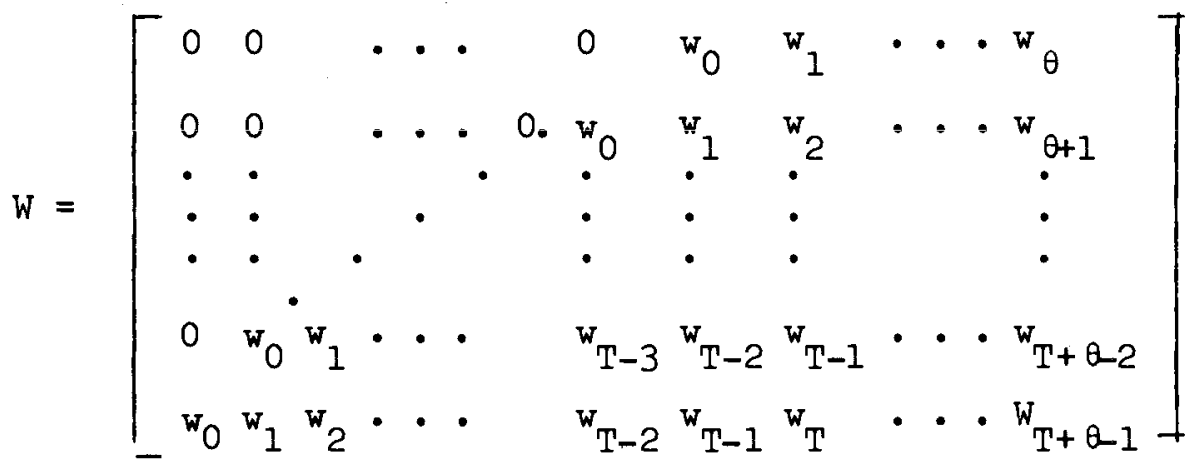

and $B$ is defined by

$$
E^{*}\left[\mathfrak{\sim}_{i}^{b} \mid{\underset{\sim}{i}}_{i}\right]=B \underset{\sim_{i}}{x_{1}}
$$

Clearly, in order to be able to identify any of the lag coefficients from $\pi^{\text {nh }}$, B must have a structure which depends on only a few unknown parameters. B will be restricted if it is reasonable to 
assume that the stochastic process generating the $x$ sequences restricts the regression of presample on insample $x$. The particular special case that we assume is given by

A3:

$$
E^{*}\left(x_{i-\theta-q} \mid x_{i}\right)=\sum_{j=1}^{p} \rho_{j}^{(q)} x_{i} j-\theta-1
$$

for $q \geq 1$; that is in the regression of the unseen past $x^{\prime} s$ on

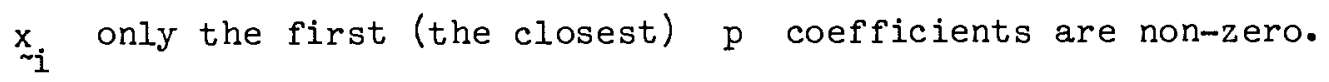
A sufficient condition for $A 3$ is that the regression of the $T+\theta-p$ most recent observations of $x$ on their entire past history depends only on their preceding $p$ values. $2 /$ This would hold, for example, if the recent $x^{\prime} s$ were a realization from a $\mathrm{p}^{\text {th }}$ order autoregressive process. It would also be true for more general processes such as an integrated autoregression (where the order of the integration plus the order of the autoregression sum to p), with possibly changing coefficients over time. $3 /$

Since each element of $\stackrel{\sim}{I}_{1}$ is just a different linear combination of the same presample $x^{\prime} s$, given $A 3$, the $B$ matrix in (2) will have the form

$$
B=[\overbrace{0}^{T+\theta-p} \overbrace{B_{p}}^{p}] .
$$

2. Mathematically the condition is that for $q \geq 1$,

$$
\begin{aligned}
& E^{*}\left[\left(x_{i T}, \cdots, x_{i-\theta+p}\right)^{\prime} \mid\left(x_{i-\theta+p-1}, \cdots, x_{i-\theta}, x_{i-\theta-q}\right)\right]= \\
& E^{*}\left[\left(x_{i T}, \cdots, x_{i-\theta+p}\right)^{\prime} \mid\left(x_{i-\theta+p-1}, \cdots, x_{i-\theta}\right)\right]
\end{aligned}
$$

3. See Anderson (1978) and the literature cited there for details on the use of such processes in mutiple time series. 
That is, $B$ may be partitioned into a $T$ by $T+\theta-p$ matrix of zeros and $a \mathrm{~T}$ by $\mathrm{p}$ matrix of free coefficients $\left(\mathrm{B}_{\mathrm{p}}\right)$. In this case then a consistent estimate of $\pi^{\text {nh }}$ yields consistent estimates of the leading $T+\theta-p$ lag coefficients without imposing any restrictions on the lag structure.

It will prove helpful to provide a more explicit solution for $\Pi^{\mathrm{nh}}$ - Noting that given $\mathrm{A} 3$

$$
E^{*}\left(b_{i t} \mid x_{i}\right)=\sum_{j=1}^{p} \phi_{t} j-\theta-1 x_{i} j-\theta-1
$$

where $\phi_{t j-\theta-1}=\sum_{q=1}^{\infty} w_{t+\theta+q} \rho_{j}(q)$ and using, for simplicity, the assumption that $p=\theta+1$, the system of regression equations defined by $E^{*}\left[y_{\sim_{i}} \mid x_{i}\right]$ has the form

$$
\begin{aligned}
& E^{*}\left[y_{i 1} \mid x_{i}\right)=\quad \sum_{j=1}^{P} \beta_{1} j-\theta-1, x_{i} j-\theta-1 \\
& E^{*}\left(y_{i 2} \mid x_{i}\right)=\quad w_{0} x_{2}+\sum_{j=1}^{p} \beta_{2} j-\theta_{-1}, x_{i j-\theta-1} \\
& E^{*}\left(y_{i 3} \mid x_{i}\right)=\quad w_{0} x_{3}+w_{1} x_{2}+\sum_{j=1}^{p} \beta_{j-\theta-1}, x_{i j-\theta-1} \\
& E^{*}\left(y_{i T} \mid x_{i}\right)=w_{0} x_{T}+\cdots+w_{T+\theta-p} x_{2}+\sum_{j=1}^{P} \beta_{T} j-\theta-1, x_{i j-\theta-1}
\end{aligned}
$$

where $\beta_{t j-\theta-1}=w_{t+\theta+1-j}+\phi_{t j-\theta-1}$, for $t=1, \ldots, T$, and $j=1, \ldots, p$. 
(4) clarifies how one identifies the leading lag coefficients from II under assumptions Al to A3. Each year's value of $y$ is regressed on current and all previous insample values of $x$. The last $p$ values of $\mathrm{x}$ in each regression equation have non-zero partial correlations with the truncation remainders ( $t$ he $b_{i t}$ ). Hence their coefficients do not identify the parameters of the lag distribution. The $t+\theta-p$ leading coefficients in each equation are, in fact, estimates of the leading lag coefficients. As $t$ increases, one gradually uncovers the lag structure. Of course, if $T+\theta$ is small relative to $p$, we will not be able to build up mach information on the tail of the lag distribution. This simply reflects the fact that short panels, by their very nature, do not contain unconstrained information on that tail. However, even when $T+\theta$ is small, the initial consistent estimates of the first few lag coefficients privided by (4) may contain enough information on the lag structure to allow one to restrict it to be a member of a family of distributions which depend on a small number of parameters and concentrate on estimating those parameters thereafter. This gets slightly more complicated when there is also heterogeneity, a topic which we turn to now.

\section{II.2 Identification With Heterogeneity}

Recall that the reason for dealing explicitly with the $\alpha_{i}$ was to allow for the possibility of unobserved individual-specific factors which cause differences in both $\mathrm{x}_{i}$ given all the individual's past 
$x^{\prime} s$, and in $x_{i} .4 /$ It follows that if there is heterogeneity, $A 3$ is not likely to provide a good approximation to the regression function of presample on insample $x$. That is, if $y$ conditional on all past $x^{\prime} s$ has an individual-specific component, and the diverse unobserved factors which lead to that component have an independent effect on insample $x^{\prime} s$, then these same factors are likely to have an independent effect on presample $x^{\prime} s$. The simplest way to incorporate this possibility into our framework is to allow the regression function of presample on insample $x$ to depend directly on the $\alpha_{i}$. That is, we would drop A2 of the last subsection (thus allowing for heterogeneity) and replace $A 3$ with the assumption

A4: $\quad E^{*}\left[x_{i-\theta-q} \mid x_{i}, D_{i}\right]=c_{q} \alpha_{i}+\sum_{j=1}^{p} \rho_{j}^{*}(q) x_{i-\theta+j-1}$, for $q \geqslant 1$. Note that $\mathrm{A} 4$ does constrain the individual-specific differences in the $x$ process to be proportional to the $\alpha_{i}$. Since this assumption may be too restrictive for many examples we develop below the generaliza-

4. If the $\alpha_{i}$ were not correlated with $\underset{\sim_{i}}{x}$ i.e., if $E^{*}\left(\alpha_{i} \mid x_{i}\right)=0$, then, given Al, the $\alpha_{i}$ would become a set of 'random' (or uncorrelated) effects in the sense of Balestra and Nerlove (1966). Since the discussion of II.1 did not restrict $E\left[\sim_{i} u_{\sim_{i}} u^{\prime} \mid x_{i}\right]$, in this case we can simply define $\tilde{u}_{i}=u_{i}+\alpha_{i}$, substitute ${\underset{\sim}{i}}_{i}$ for ${\underset{\sim}{i}}_{i}$ in subsection II.l, and apply the results obtained there. 
tions required when we replace the $\alpha_{i}$ in $A_{4}$ with a separate unobserved individual specific component, say $m_{i} . \underline{5 /}$

Let $\tilde{x}_{i t}=\left(x_{i t}-a_{t} m_{i}\right)$ for $i=1, \ldots, N$, all $t$, and some sequence $\left\{a_{t}\right\}$. Then if $\tilde{x}_{i t}$ is substituted for $x_{i t}$ in the discussion of the sufficient conditions for A2 (see p.9) those conditions become sufficient for A4. Familiar examples of processes which satisfy this condition are processes in which, for $t=-\theta+p, \cdots, T$, $x_{i t}=\gamma^{t} \alpha_{i}+\sum_{\tau=1}^{p} \kappa_{\tau}^{t} x_{i t-\tau}+\xi_{i t}$, where $E^{*}\left[\xi_{i t} \mid D_{i}, x_{i t-1}, x_{i t-2}, \cdots\right]=0$. With $\gamma^{t}=\gamma$ and $k_{\tau}^{t}=\kappa_{\tau}$ for $t=-\theta+p, \ldots, T$, this is a mixture process consisting of an individual-specific component and an integrated autoregressive deviate whose coefficients are stable over time (MaCurdy, 1982, gives several examples of the use of such processes).

Using $A 4$ to find $E^{*}\left(b_{i} \mid D_{i}, \underset{\sim_{i}}{x_{1}}\right)$ and substituting the result into Al yields

5. A simple example where the restriction is relevant is provided by Mundlak (1961). In that article, the problem is to estimate a production function from panel data on the inputs $(x)$ and output (y) of a group of firms operating in a competitive market. In this case the $\alpha_{i}$ represent unobserved interfirm productivity differences that are constant over time and known to management at the time input decisions are made. 


$$
\begin{aligned}
& E^{*}\left(y_{i t} \mid x_{i}, D_{i}\right) \\
& =c_{t}{ }_{i}+\sum_{\tau=0}^{t+\theta-p}{ }^{t}{ }_{\tau} x_{i} t-\tau+\sum_{j=1}^{P} \beta_{t}^{*} j-\theta-1 x_{i j-\theta-1}
\end{aligned}
$$

where $c_{t}=\left[1+\sum_{q=1}^{\infty} w_{t+\theta+q} c_{q}\right]$, and $\beta_{t}^{*} j-\theta-1=w_{t+\theta+1-j}+\sum_{q=1}^{\infty} w_{t+\theta+q} \rho_{j}^{*(q)}$, for $t=1, \ldots, T$, and $j=1, \ldots, p$. Note that in equation (5) the $\alpha_{i}$ have different coefficients in the different years of the sample. This follows from the fact that the truncation remainders are different linear combinations of the presample $x^{\prime} s$. If $w_{\tau}=0$ for $\tau>T+\theta$, that is, if the length of the lag is less than or equal to $\mathrm{T}+\theta$, then there is no truncation remainder in period $\mathrm{T}$, and $c_{T}=1$. In the general case, however, equation (5) has a single unobserved factor with free 'factor loadings' (the $c_{t}$ ). Since the contribution of the $\alpha_{i}$ in year $t$ can only be identified relative to its contribution in some other year, a normalization of the factor loadings is required and one which is consistent with the possibility that the length of the lag is less than or equal to $T+\theta$ is $c_{T}=1$. To show which lag coefficients are identified in this case we rewrite (5) as

$$
y_{i t}-\tau_{t} y_{i 1}=\sum_{\tau=0}^{t-1} w_{\tau} x_{i t-\tau}+v_{i t}
$$

for $i=1, \ldots, N$ and $t=2, \ldots, T$, where $\bar{c}_{t}=c_{t} / c_{1}$, and $v_{i t}=y_{i t}-c_{t} y_{i l}-E^{*}\left(y_{i t}-c_{t} y_{i t-1} \mid x_{i}, D_{i}\right)$, so that $E^{*}\left(v_{i} \mid x_{i}, D_{i}\right)=0$ by construction, and we have assumed for simplicity that $\theta+1=p$. This is a standard simultaneous equation system and will identify the leading $T+\theta-p$ lag coefficients (if 
$\theta+1=p, \quad T+\theta-p=T-1$ ) if there is an equation (year) in which at least one $x$ is excluded from the determinants of $\mathrm{y}_{i t}-\bar{c}_{t} \mathrm{y}_{i l}$ while still being correlated with $\mathrm{y}_{i l}$. If $\mathrm{T} \geq 3$, then $x_{i 3}, x_{i 4}, \ldots, x_{i T}$ are excluded from the equation determining $\mathrm{y}_{i 2}-\bar{c}_{2} \mathrm{y}_{i 1}$, and provided they have a non-zero partial correlation with the $\alpha_{i}$, they will be correlated with $y_{i l}$.

To find the $I I$ matrix corresponding to (5) (that is, the matrix under assumptions $A l$ and $A 4$ which we denote by $\pi^{\text {sh }}$ ), we use the fact that $E^{*}\left(x_{i} \mid x_{i}\right)=E^{*}\left\{\left(E^{*}\left(y_{r_{i}} \mid x_{i}, D_{i}\right) \mid x_{i}\right\}\right.$, to derive

$$
\pi^{S h}=W+B+\underset{\sim}{c} \underline{\sim}^{\prime}
$$

where $W$ and $B$ are defined in (2) and $(3), \underset{\sim}{c^{\prime}}=\left[1, c_{T-1}, \ldots, c_{1}\right]$, and $\alpha_{i}$ denotes the vector of coefficients resulting from the regression of $\alpha_{i}$ on ${\underset{\sim}{i}}_{i}$ li.e., $\left.E^{*}\left(\alpha_{i} \mid x_{i}\right)=\sum_{r=-\theta}^{T} \underset{r}{\alpha} x_{i r}\right) \cdot \operatorname{Recall}$ that $\pi^{n h}=W+B$ so that the difference in the $\Pi$ matrices that results from allowing for the $\alpha_{i}$ (heterogeneity) is just $\underset{\sim}{c} \alpha^{\prime}$, the matrix of coefficients obtained from the regression of $\underset{\sim}{c} \alpha_{i}$ on $x_{i}$

If one were to allow for two different sources of heterogeneity, one in the distributed lag model (the $\alpha_{i}$ ) and one in the process generating the $x^{\prime} s$ (the $m_{i}$ ), then to identify the leading lag coefficients we would require $T \geq 4$, and the $\Pi$ matrix (which in this case we denote by $\pi^{\text {th }}$ ) would be given by

$$
\Pi^{\text {th }}=W+B+{\underset{\sim}{c}}^{*} \stackrel{m}{\prime}^{\prime}+\underset{\sim}{\ell \alpha^{\prime}}
$$


where $\mathrm{W}, \mathrm{B}$, and $\underset{\sim}{\alpha}$ are defined as in $(6), \underset{\sim}{c^{*}}=\left[1, c_{1}^{*}, \ldots, c_{\mathrm{T}}^{*}\right]$, $\underset{\sim}{m}$ is the vector of coefficients obtained from the regression of $m_{i}$ on $\underset{\sim i}{x_{i}} \quad$ [i.e. $\left.E^{*}\left(m_{i} \mid x_{i}\right)=\sum_{r=-\theta}^{T} m_{r} x_{i r}\right]$, and $\underset{\sim}{\ell}$ here, and in the discussion below, is a vector of ones. Note that when two sources of heterogeneity are allowed for it is $m_{i}$ which has a variable coefficient over time, and if the length of the lag is less than $T+\theta$, its coefficient in year $T$ is zero. To allow for this possibility the normalization, $c_{1}^{*}=1$ is used (instead of $c_{T}=1$ as in $\left.\pi^{\mathrm{sh}}\right) .6 /$

II.3 Some Further Considerations: Estimation and Testing

Having discussed the identification of the lag parameters under alternative assumptions, we turn now to the consideration of some issues associated with the choice of estimators and test statistics for such models.

In the context of panel data one is interested in the limiting properties of estimators as $\mathrm{N}$ (the number of individuals) grows large, and these depend on how the sample moments behave as the sample grows in this dimension. Chamberlain (1982) provides an explicit discussion of the properties of alternative estimators of $\Pi$ and of relevant test statistics under the assumption that the $\mathrm{N}$ obser-

6. With two sources of heterogeneity there is an additional normalization to be imposed on one of the elements in $\underset{\sim}{\alpha}$ or in $\underset{\sim}{\mathrm{m}}$. 
vations on the vector ${\underset{\sim}{\sim_{i}}}^{\prime}=\left(\mathrm{x}_{\sim_{1}}{ }^{\prime},{\underset{\sim}{i}}^{\prime}\right)$ are independent draws from some multivariate distribution with finite fourth order moments. Note that neither this assumption, nor any of the assumptions made prior to it, imply that $\left.E\left\{y_{\sim i}-\Pi_{\sim i}^{\prime}\right)\left(y_{\sim i}-\Pi_{\sim_{i}}\right)^{\prime} \mid x_{\sim_{i}}\right\}=\Omega$ (independent of i ) Thus random coefficients models, and models in which there is a complicated non-linear relationship between the $\alpha_{i}$ (or $m_{i}$ ) and ${\underset{\sim}{i}}_{i}\left[\right.$ i.e. $\left.E^{*}\left(\alpha_{i} \mid x_{i}\right) \neq E\left(\alpha_{i} \mid x_{i}\right)\right]$ do not necessarily violate any of our assumptions (for a discussion of related points and alternative stochastic assumptions, see, in addition, White, 1980, 1980a, 1980b, and 1982). With these assumptions maximum likelihood (based on a normal density for $\underset{\sim i}{r}$ ) or an asymptotically equivalent estimator of $I$ is (strongly) consistent and asymptotically normal but need not be efficient, nor need its covariance matrix be given by the traditional information matrix (see also MaCurdy, 1981). The point to note here is that unless further assumptions on the covariance matrix of disturbances from the regression of $y_{i}$ on $\underset{\sim_{i}}{x}$ are relevant, one mist take care in formulating standard errors and test statistics from maximum likelihood estimation procedures (Chamberlain, 1982, provides both the appropriate formulae and more efficient estimators, under these assumptions). Of course, if $E\left\{\left(x_{i}-\Pi_{x_{i}}\right)\left(y_{\sim_{i}}-\Pi_{x_{i}}\right) \mid x_{i}\right\}=\Omega$ then maximum likelihood is efficient, and variances and test 
statistics are formed in the usual manner. I'

Looking at the structure of the II matrices, it is clear that for $\mathrm{t} \geq 4, \pi^{\mathrm{th}}$ can be obtained from a set of restrictions on $\pi$, $\pi^{\text {sh }}$ from restrictions on $\pi^{\text {th }}$, and $\pi^{\text {th }}$ from restrictions on $\pi^{\text {sh }}$. There is, therefore, a nested sequence of restrictions that can be used to decide between alternative models. In particular, if there is no heterogeneity, then the elements of $\mathrm{II}=\left[\pi_{\mathrm{tr}}\right]$ ought to be close to zero for $r>t \geq p-\theta$ (that is all leading $x^{\prime} s$ for years where $t \geq p-\theta$ should have insignificant coefficients); and its elements should have a "stationary structure" (should depend only on $t-r)$ for $t \geq r>p-\theta$. The test for a model with no heterogeneity involves, therefore, only equality and zero restrictions.

7. Note that our discussion is based on estimating the $I$ matrix rather than on estimating the form of the model containing either or both of the sequences $\left\{\alpha_{i}\right\}_{i=1}^{N}$ and $\left\{m_{i}\right\}_{i=1}^{N}$ (the system in equation (5) for example). Since they affect the distribution of only $T$ (a small number) of sample points, one cannot estimate them consistently as $\mathrm{N} \rightarrow \infty$ and an attempt to do so would lead, in general, to inconsistent estimates of the other parameters of interest. That is, in our problem $\left\{\alpha_{i}\right\}_{i=1}^{N}$ and $\left\{m_{i}\right\}_{i=1}^{N}$ are sets of incidental parameters in the sense of Neyman and Scott (1948). By going directly to the $I I$ matrix we are, in fact, constructing the density of $\mathrm{y}_{\sim i}$ conditionals on $\underset{\sim_{i}}{\mathrm{x}_{1}}$, and the structural parameters of the model (i.e. the parameters governing the distributions of each $\underset{\sim i}{r}$ ), but marginal to the model's incidental parameters; see Kiefer and Wolfowitz (1956). 
This assumes that $p$ is known at the outset. If this is not the case then one can build a nested testing sequence for $p$ by starting with p sufficiently large and testing the constraints on $\Pi$ arising from lower values of $p$. In all cases the test of the null hypothesis that $\mathrm{p}=\mathrm{p}^{*}-1$ against the alternative that $\mathrm{p}=\mathrm{p}^{*}$ consists of testing whether the left row of the submatrix $\mathrm{B}_{\mathrm{p}}^{*}$ (see equation (3)) is a vector of zeroes. Provided $\mathrm{p}^{*} \geq \theta+1$, the null hypothesis imposes $T-1$ additional constraints on the $\pi$ matrix.

Note also that if we denote the matrix of second order population moments

$$
\Sigma_{r r^{\prime}}=\left[\begin{array}{cc}
\Sigma_{x x^{\prime}} & \Sigma_{x y^{\prime}} \\
& \Sigma_{y y^{\prime}}
\end{array}\right],
$$

8. The choice of rejection criteria for such a sequence of tests is discussed in Geweke and Meese (1979). Their discussion is likely to be particularly relevant when $p$ can take on a wide range of values (so that a long sequence of tests may be relevant). In our case, one is likely to have fairly narrow a priori bounds on $p$. The assumptions in the text constrain only II - If, however, one were willing to assume that the leads which receive zero coefficients in the projection of past $x^{\prime} s$ on future $x^{\prime} s$, (or on future $x^{\prime} s$ and $D_{i}$ ) are independent of time, then one could test A2 (A4) directly by considering the regression of early observed $x^{\prime} s$ on later ones (or on the later ones and $D_{i}$, and the projection of $\mathrm{D}_{i}$ onto $\underset{\sim_{i}}{x_{i}}$ ). The procedure should prove helpful in suggesting a relevant range for $p$. 
then the only constraints our assumptions place on $\Sigma_{r r^{\prime}}$, are constraints on $\mathrm{II}^{\prime}=\left[\Sigma_{\mathrm{xx}}\right]^{-1} \Sigma_{\mathrm{x} \mathrm{y}^{\prime}} ;$ a matrix which also contains all the parameters of interest. Assumption A2 or A4 does impose an addiadditional set of constraints on moments from the joint distribution of presample and insample $x$, but the effect of either assumption on $\Sigma_{r r^{\prime}}$, is limited to its effect on $\Pi$. However, if one can assume that the process generating $x_{i}$ is one of the simpler stochastic prom cesses that satisfies $A 2$ or $\mathrm{A} 4$ ), then the constraints are in terins of the elements of both $\Pi$ and $\Sigma_{x x^{\prime}}$. In this case more efficient estimates of the lag coefficients and more powerful test statistics can be obtained by imposing the constraints on both of these matrices. For example, if $x_{i t}=m_{i}+z_{i t}$, where $z_{i t}$ is a $p^{\text {th }}$ order autoregressive deviate with partial correlations $\rho_{1}, \ldots, \rho_{p}$ and variance $\sigma^{2}$ for $i=1, \ldots, N$ and all $t$ (this is a simple mixture process satisfying $\left.A^{4}\right)$, then $\sum_{x x^{\prime}}=E\left[{\underset{\sim}{x}}_{\sim_{i}^{\prime}}^{\left.x_{i}^{\prime}\right]}=\sigma_{m}^{2} \underset{\sim}{\ell \ell^{\prime}}+\Sigma^{*}\right.$, where $\sigma_{m}^{2}=E m_{j}^{2}$, and $\Sigma^{*}$ is the covariance matrix for $T+\theta$ consecutive observations on a $\mathrm{p}^{\text {th }}$ order autoregressive deviate (formulae for the elements of $\Sigma^{*}$ in terms of the parameters $\rho_{1}, \ldots, \rho_{p}$ and $\sigma^{2}$ are provided in Galbraith and Galbraith, 1974). Clearly then the $(T+\theta) \cdot$ $(T+\theta+1) / 2$ elements of $\Sigma_{x x^{\prime}}$ are a function of only $p+2$ 
parameters. Moreover, these same parameters determine some of the elements of $\pi^{\text {th }}$ (or $\pi^{\text {sh }}$ )..$^{9}$

Finally, though our discussion has been concerned with models which contain only a single regressor it is not difficult to extend our results to cases where there are more than one of them. In this case there would be a vector of $x^{\prime} s$ for each individual in every

9. To see this note that in equation (7), $\underset{\sim}{m}=\Sigma_{x x^{\prime}}^{-1} \underset{\sim}{\stackrel{2}{a}}$. It is straightforward to verify that $\Sigma_{x x^{\prime}}^{-1}=\Sigma^{*-1}-\theta_{1} \Sigma^{*-1} \underset{\sim \sim}{\underset{\sim}{\ell}} \Sigma^{*-1}$ where $\theta_{1}=\sigma_{m}^{2} /\left(1+\underset{\sim}{\ell} \Sigma^{*-1} \underset{\sim}{\ell} \sigma_{m}^{2}\right)$. Thus $m_{\sim}=\Sigma^{*-1} \underset{\sim}{\ell} \theta_{1}$, which is a function of the same parameters as $\Sigma_{x_{x^{\prime}}}$ (in fact $\mathrm{m}_{\mathrm{r}}=\mathrm{m}$ for $\mathrm{T}-\mathrm{p}>\mathrm{r}>\mathrm{p}-\theta$, while $\mathrm{m}_{-\theta+j}=\mathrm{m}_{\mathrm{T}-\mathrm{j}}$ for $0 \leq j \leq p)$. Finally the $c_{q}$ and $\rho_{j}^{(q)}$ coefficients in $A^{4}$ will be determined from the backcasting function for this process. In particular the $\rho_{j}^{(q)}$ will follow the recursion formulae for backcasting coefficients from autoregressive processes $\left(\rho^{(q+1)}=\rho_{j} \rho_{1}^{(q)}+\rho_{j+1}^{(q)}\right.$, where it is understood that $\rho_{j}^{(\cdot)}=0$ for $j>p$ and $\rho_{j}^{(1)}=\rho_{j}$ for $j=1, \ldots, p$; see Box and Jenkins, 1970), and $c_{q}=1-\sum_{j=1}^{P} \rho(q) . ~ G i v e n$ the definition of $c$ [equation (5)], and of $B$ and $W$ lequation (3)), these formulae provide an additional set of non-linear constraints connecting the elements of $\pi$ to those of $\Sigma_{x x^{\prime}}$. 
period, say, $x_{i t}$, and one requires assumptions on the regression

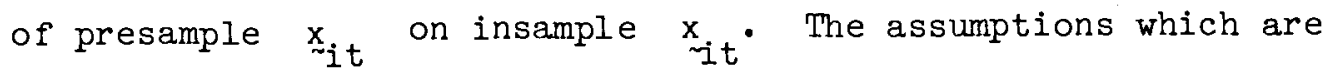
analogous to $\mathrm{A} 2$ and $\mathrm{A} 4$ are, respectively,

$E^{*}\left[x_{i}-\theta-q \mid x_{i-\theta}, \ldots, x_{i T}\right]=E^{*}\left|x_{i-\theta-q}\right| x_{i-\theta}, \ldots, x_{i-\theta+p} \mid$ and $E^{*}\left[x_{i}-\theta-q\left|x_{i}-\theta, \cdots, x_{i T}, D_{i}\right|=E^{*}\left|x_{i}-\theta-q\right| x_{i}-\theta, \ldots, x_{i}-\theta-p, D_{i}\right]$ for $q \geq 1$. These would be satisfied, for example, by a time inhomogeneous $\mathrm{p}^{\text {th }}$ order vector autoregression, and by a mixture process consisting of individual specific components (one for each regressor) and a $\mathrm{p}^{\mathrm{th}}$ order vector autoregression. $10 /$ In either case the II matrix would allow for the coefficients of the earliest $p$ values of each regressor in every year to be unconstrained regardless of how many of the regressors had a distributed lag effect on $y$.

III. Distributed Lags from Panel Data with Prior Structure on the Lag Coefficients

As noted earlier in order to identify any of the lag coefficients from panel data one must impose some structure on the relationship between the truncation remainders and the observed data. The last section discussed the possibility of restricting the relationship between presample and insample $\mathrm{x}$; in this section we consider restricting the lag coefficients themselves. The particular example we focus on is a sequence of lag coefficients which, after a few free lags, has an auto-

10. Kiefer, 1979, discusses the problem of determining whether a smaller number of individual-specific components would suffice. 
regressive structure [such structures have been widely used the past; see, e.g., Dhrymes (1971), Griliches (1967) and Nerlove (1972)]. This restriction is formalized in the assumption

A5: $\quad \mathrm{w}_{\tau}= \begin{cases}{ }_{\tau} & \text { for } \tau \leq \mathrm{k}_{1} \\ \sum_{\mathrm{q}=1}^{\mathrm{Q}} \delta_{\mathrm{q}}{ }^{\mathrm{w}}{ }_{\tau-\mathrm{q}} & \text { otherwise }\end{cases}$

where the roots of the characteristic equation $1-\sum_{q=1}^{Q} \delta_{q} z^{q}=0$, say $\lambda_{1}^{-1} \ldots, \underset{q}{\lambda^{-1}}$ lie outside the unit circle. For simplicity we assume that $\mathrm{k}_{1}=\theta$, and that $\lambda_{1}, \ldots, \lambda_{Q}$ are all real and distinct.

If $\mathrm{A} 5$ is substituted into Al the set of truncation remainders from the resulting distributed lag model follow an exact $Q^{\text {th }}$ order autoregression. They can, therefore, be written as $b_{i t}=\sum_{j=1}^{Q} \lambda_{j}^{t} b_{i j}$ where $b_{i j}=w_{\theta-j+1} \sum_{\tau=1}^{\infty} \lambda_{j}^{\tau} x_{i-\tau-\theta}$; that is, $b_{i l}, \ldots, b_{i Q}$ are a set of $Q$ unobserved initial conditions. Under $A 5$, then, the distributed lag model Al becomes a system of $T$ regressions with $Q+1$ freely correlated unobserved factors $\left(\alpha_{i}, b_{i l}, \ldots, b_{i Q}\right), Q$ of them having factor loadings which decay geometrically over time. Since the identification properties of a model in which there are $Q+1$ unobserved factors are a straightforward generalization of a model with two of them, we deal first with the familiar case of a (modified) geometric or Koyck lag in which $Q=1$, and then point out the modifications required for $Q>1$.

Setting $Q=1$ into $A 5$, and substituting the result into $A l$, we have: 


$$
\begin{aligned}
& y_{i t}=\alpha_{i}+\sum_{\tau=0}^{\theta} w_{\tau i t-\tau} x_{i}+w_{\theta} \sum_{\tau=\theta+1}^{t+\theta} \delta^{\tau-\theta} x_{i t-\tau} \\
& +\delta^{t-1} b_{i}+u_{i t}
\end{aligned}
$$

where $b_{i}=w_{\theta} \sum_{\tau=1}^{\infty} \delta^{\tau} x_{i-\tau-\theta}$, that is, $b_{i}$ is the truncation

remainder in period one (and the initial condition when $Q=1$ ) for $i=1, \ldots, N$ and $t=1, \ldots, T$.

Given the discussion of the last section it is not difficult to see why the lag coefficients in (8) are identified from the $\pi$ matrix. Recall that to identify the lag parameters one requires a set of restrictions on the matrix $B=\left[\beta_{t r}\right]$, where $E^{*}\left[b_{i} \mid x_{i}\right]=B_{i}$. Given our assumptions $b_{i t}=\delta b_{i t-1}$, so that $E^{*}\left[b_{i t} \mid x_{i}\right]=$ $\delta E^{*}\left[b_{i} t-1 \mid x_{i}\right]$ which implies that $\beta_{t r}=\delta \beta_{t-1} r$ for $r=1, \ldots, T+\theta$ and $t=2, \ldots, T . \underline{11 /}$

11. Note the difference here between the estimates of (modified) geometric lag structures from panel data and those from a single time series. In the latter case the relevant limiting dimension is $T$, and as $T$ gets larger the dependence of consistent estimators of the lag coefficients on the value chosen for $b_{i}$ generally disappears (Dhrymes, 1971). When $T$ is short this result no longer applies. In particular with large $N$ as in panel data we have a set of truncation remainders and the properties of their distribution (i.e., $E^{*}\left(b_{i} \mid x_{i}\right)$ ) do affect the properties of alternative estimates of the lag coefficients (see also Pesaren, 1973). 
It follows that the $\pi$ matrix (which in this case we denote by $\pi^{f}$ ) has the form:

$$
I^{g}=W^{*}+\underset{\sim}{\delta} \underline{\sim}^{\prime}+\underset{\sim}{i}{\underset{\sim}{\prime}}^{\prime}
$$

where $\underset{\sim}{i}$ and $\underset{\sim}{\alpha}$ are defined as in $(7), \underset{\sim}{\delta}=\left[1, \delta^{\prime}, \ldots, \delta^{T}\right\}, \underset{\sim}{\beta}$ is the vector of coefficients from the regression of $b_{i}$ on $x_{i}$ [i.e., $\left.E^{*}\left(b_{i} \mid{\underset{\sim}{x}}_{i}\right)=\sum_{r=-\theta}^{T} \beta_{r} x_{i r}\right]$ and

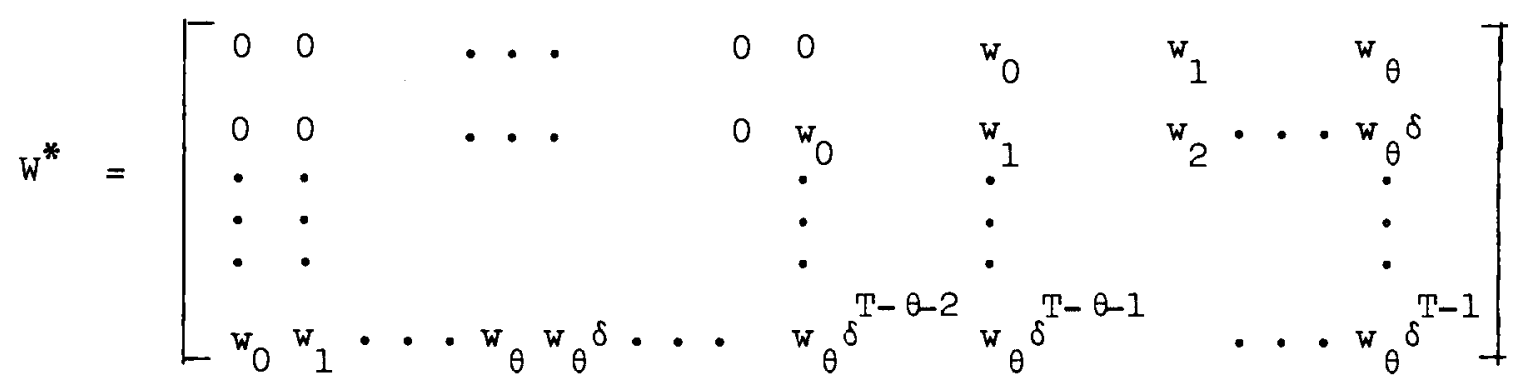

$\Pi^{8}$ will identify the lag coefficients provided $\mathrm{T} \geq 3 . \underline{12 /}$ In the more general case with $Q>1$ one would replace $\underset{\sim}{\delta B^{\prime}}$ in (9) by

12. To see this note that (8) implies that

$$
\begin{aligned}
& \left(y_{i t}-y_{i t-1}\right)-\delta\left(y_{i t-1}-y_{i t-2}\right)=w_{0} x_{i t}+\left[w_{1}-w_{0}(1-\delta)\right] x_{i t-1} \\
& +\sum_{\tau=2}^{\theta}\left[w_{\tau}-(1-\delta)_{w-1}+w_{\tau-2}\right] x_{i t-\tau}+v_{i t}
\end{aligned}
$$

where $E^{*}\left[v_{v_{i}} \mid x_{i}\right]=0$ for $i=1, \ldots, N$ and $t=2, \ldots, T$. In this form we have a standard simultaneous equations system. Provided $T \geq 3, x_{i 3}, \ldots, x_{i T}$ will serve as instruments for the equation determining $\mathrm{y}_{i 2}-\mathrm{y}_{\mathrm{il}}$. 
$\sum_{j=1}^{Q} \underset{\sim j}{\beta_{j}^{\prime}}$, and the $\Pi$ matrix would identify the lag coefficients if $\mathrm{T} \geq \mathrm{Q}+2$

One further point is worthy of note. In this section we have not restricted the relationship between presample and insample $x$ in any way, and as a result the $I$ matrix resulting from assumptions $A I$ and A5 are not, in general, nested inside the $I$ matrices of Section II. If, however, in addition to $A 5$ we assume $A 2$ or $A 4$ of the last section, then the $I$ matrices from the models in this section can be simplified further (the additional restrictions are straightforward special cases of the restrictions developed in Section II) and the models presented here will be nested to those presented in the last section. Thus, if either A2 or A4 were relevant, and the initial consistent estimates of a free lag distribution obtained from the techniques developed in the last section indicated that one of the simple lag structures satisfying A5 seemed plausible, one could test and then estimate this lag structure in a second round.

IV. AN EXAMPLE: ESTIMATING THE SHAPE OF THE DEPRECIATION FUNCTION

A common empirical construct is the "stock of capital" based on some variant of the perpetual inventory method. It is, in fact, a distributed lag function of past investment expenditure with the depreciation formula implying a set of lag weights (w's). These weights are, usually, assumed a priori based on mortality assumptions (straight line, geometric, etc.) and some scattered evidence on the length of life of selected assets. Alternatively, one accepts the 
depreciation assumptions used by firms and reported in their annual statements, even though they may be heavily affected by tax and other accounting considerations that have little to do with the economic measure of depreciation one is after. In general, there is no obvious unique right way to construct "capital"; it all depends on the purpose for which such a variable is to be constructed (Cf Griliches 1963, and the articles in Usher, ed. 1980).

One of the more common uses for capital is to compute and compare "rates of return" across projects, firms, or industries. Such measures assume implicitly that there exists a stable relationship between earnings (gross or net profits) and past investments, and that firms or industries differ only by a factor of proportionality in the yield on their past investments, with the time shape of these yields being the same across firms and implicit in the assumed depreciation formula. In this section we investigate this question empirically, using panel data and the methods developed in Section II to estimate an unconstrained distrubuted lag relationship between firm profits and their past investments.

The data come from the Compustat tape (1980). Profits are for the years 1964-72 inclusive (thus $T=9$ ) and are defined to equal the operating income of firms deflated by the implicit G.N.P. deflator and an index of the average gross rate of return $(1972=1.0)$ taken from Feldstein and Summers (1977, table 1, column 3, page 216). The observed investment data are for the years 1961-71 (thus, assuming that this year's investment does not effect this years profits, we have two presample observations on investment). Investment is defined to equal the reported gross investment of firms deflated by the impli- 
cit G.N.P. investment deflator. Firms were chosen from the tape if there was no evidence of a major merger, acquisition, or sale of a part of the firm during the sample period; and if all the required variables for the firm were available. This yielded a sample (N) of 258 firms. $13 /$

All results reported here are based on two simplifications. First, after making an adjustment for heteroskedasticity, we shall assume that the conditional (on ${\underset{\sim}{i}}_{j}$ ) covariance matrix of disturbances from the regression of $y_{\sim i}$ on $x_{i}$ does not vary over observations. This implies that maximum likelihood estimation procedures provide consistent estimates of all parameters and their standard errors, and maximum likelihood is used throughout. The adjustment made for heteroskedasticity was to divide all figures for each firm by the square root of the average rate of investment over the 1961-71 period as a whole. The second simplification to be used here concerns the relationship between presample and insample $x$. Our results focus on the model which allows for heterogeneity and, therefore, assumption $\mathrm{A} 4$ will be used to describe this relationship. It will be assumed that $\mathrm{p}$ in $\mathrm{A} 4$ equals three. This is based on a preliminary examination of the observed $x$ sequences (see note 8) which indicated that a $\mathrm{p}$ of 2 or 3 would do.

13. It also, no doubt, resulted in some selection bias. The estimates presented here do, however, allow for firm effects or heterogeneity, and this should take care of most of the sample selectivity problem. 
The Compustat tape for the years of interest contains more information on the history of the firms $x$ 's than just the reported investment series. It reports also the firm's gross $\left(k^{g}\right)$ and net $\left(\mathrm{k}^{\mathrm{n}}\right)$ capital stocks at the beginning of each year. These numbers are essentially linear combinations of the firm's past investment expenditures (including investments in years that we do not have in our data). Net capital makes adjustments for reported depreciation expenses while $\mathrm{k}^{\mathrm{g}}$ does not. ${ }^{14 /}$ The availability of $\mathrm{k}^{\mathrm{n}}$ allows us to make comparisons between the results from the models based on the techniques developed in this paper and results that would be obtained if one assumed Al but constrained the lag coeffiecients such that $k_{i t}^{n}=\sum_{\tau=0}^{\infty} w_{\tau} x_{i t-\tau}$, (a model which assumed that firms report the relevant measure of capital stock). To build the comparison model, note that under the latter assumptions $E^{*}\left[y_{i t} \mid \underset{\sim i}{k_{i}}, D_{i}\right]=\alpha_{i}+a_{1} k_{i t}^{n}$ and, $E^{*}\left[\begin{array}{ll}\alpha_{i} & k_{i}^{n}\end{array}\right]=a_{2} k_{i=}^{n}$, so that

14. Though there are some additional adjustments, the basic recursion formulae for $k^{g}$ and $k^{n}$ are: $k_{i t}^{g}=k_{i t-1}^{g}+x_{i t-1}-d_{t-1}$ where dis $_{t-1}=$ discards in period $t-1$, and; $k_{i t}^{n}=k_{i t-1}^{n}+x_{i t-1}-d_{t-1}-\operatorname{dep}_{t-1}$, where $\operatorname{dep}_{t-1}$ is the firms reported depreciation in period $t-1$. Depreciation is that part of past investments written off in the given year. The precise formulae for the fraction of each year's investment that is written of in subsequent years differ among firms, the most frequently used being a straight line depreciation scheme. 


$$
y_{i t}=a_{1} k_{i t}^{n}+a_{2} k_{i}^{n}+v_{i t}
$$

where $E^{*}\left[v_{i} \mid k_{j}^{n}\right]=0$, and $k_{i}^{n}$ is each firm's mean $k_{i t}^{n}$ over the sample period, for $i=1, \ldots, N, t=1, \ldots, T$.

Here we shall also assume that $E\left[v_{\sim_{i}} v_{\sim l}^{\prime} \mid k_{i}^{n}\right]=\Omega_{k}$, and use maximum likelihood estimation techniques. Note that the model in (10) is not nested to the model in Section II which allows for a single source of heterogeneity. In particular though $\mathrm{k}_{\mathrm{it}}^{\mathrm{n}}$ imposes the particular lag structure implicit in the Compustat calculations, it also uses information on (presample) $x^{\prime} s$ not used in the models of the last section.

We shall also make use of the information contained in $\mathrm{k}^{\mathrm{g}}$. Note that if $k_{i-\theta}^{g}=\sum_{\tau=1}^{\infty} f_{\tau} x_{i-\theta-\tau}$, and the model with a single source of heterogeneity is used then that model ( $A 4$ ) implies that

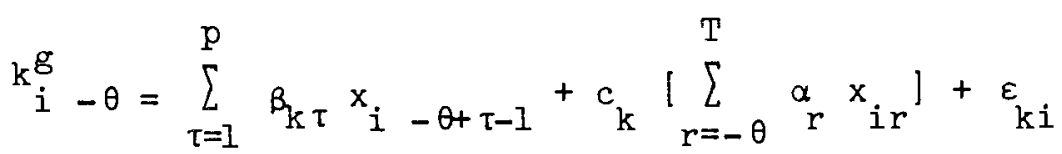

where $E^{*}\left[\varepsilon_{k i} \mid x_{i}\right]=0$, for $i=1, \ldots, N$. If we assume that $E\left[\varepsilon_{k i}^{2} \mid x_{i}\right]=\sigma_{k}^{2}$, and allow $\varepsilon_{k i}$ to be freely corrected with $\varepsilon_{i}=y_{i}-\underset{\sim_{i}}{I x}$, then equation (11) can be added to the system in (6), to produce a system with $\mathrm{T}+1$ equations, which, since it uses the information on the presample $x^{\prime} s$ contained in $k^{g}$, should provide more precise parameter estimates than one could derive from using (6) alone. 
Table 1 summarizes the parameter estimates. Estimates of the unconstrained $I$ matrix indicated that there was heterogeneity (see p.18). Column (1) lists the estimated lag coefficients based on allowing for a single source of heterogeneity (the model of assumptions $\mathrm{Al}$ and $\mathrm{A}_{4}$, or the $\Pi$ matrix in (6)) while column 2 adds equation (11) to this system. The algorithm used to compute the maximum likelihood estimates of column (1) did not actually meet its convergence criteria. It seemed that the data could not distinguish effectively between alternative combinations of the vectors $\underset{\sim}{\alpha}$ and $\underset{\sim}{c}$ and the last few lag coefficients when using system (6); but convergence was rapid once we added equation (11). The resulting estimates, listed in column (2), yield $w_{1}$ through $w_{8}$ which are all positive, the first seven being estinated with a fair amount of precision. As one should expect from the structure of the $\Pi$ matrix, the standard errors of the lag coefficients rise monotonically in $\tau$. While it is clear that the lag coefficients increase until $\tau=3 ; w_{3}, w_{4}, w_{5}$, $w_{6}, w_{7}$ and $w_{8}$ are not very different from each other (though there is weak evidence of a slight fall in the values of the last one, perhaps two, coefficients). The observed value of the $x_{4}^{2}$ test statistic of the null hypothesis that $w_{3}=w_{4}=w_{5}=w_{6}=w_{7}=w$ was 2.05 which is below the expected of a $x_{4}^{2}$ deviate, and imposing this restriction produced the estimates of column (3). A test of the additional constraint that $w_{8}=w$, produced an observed $\chi_{1}^{2}$ test statistic of 1.91 (still not significant at usual levels of significance), 


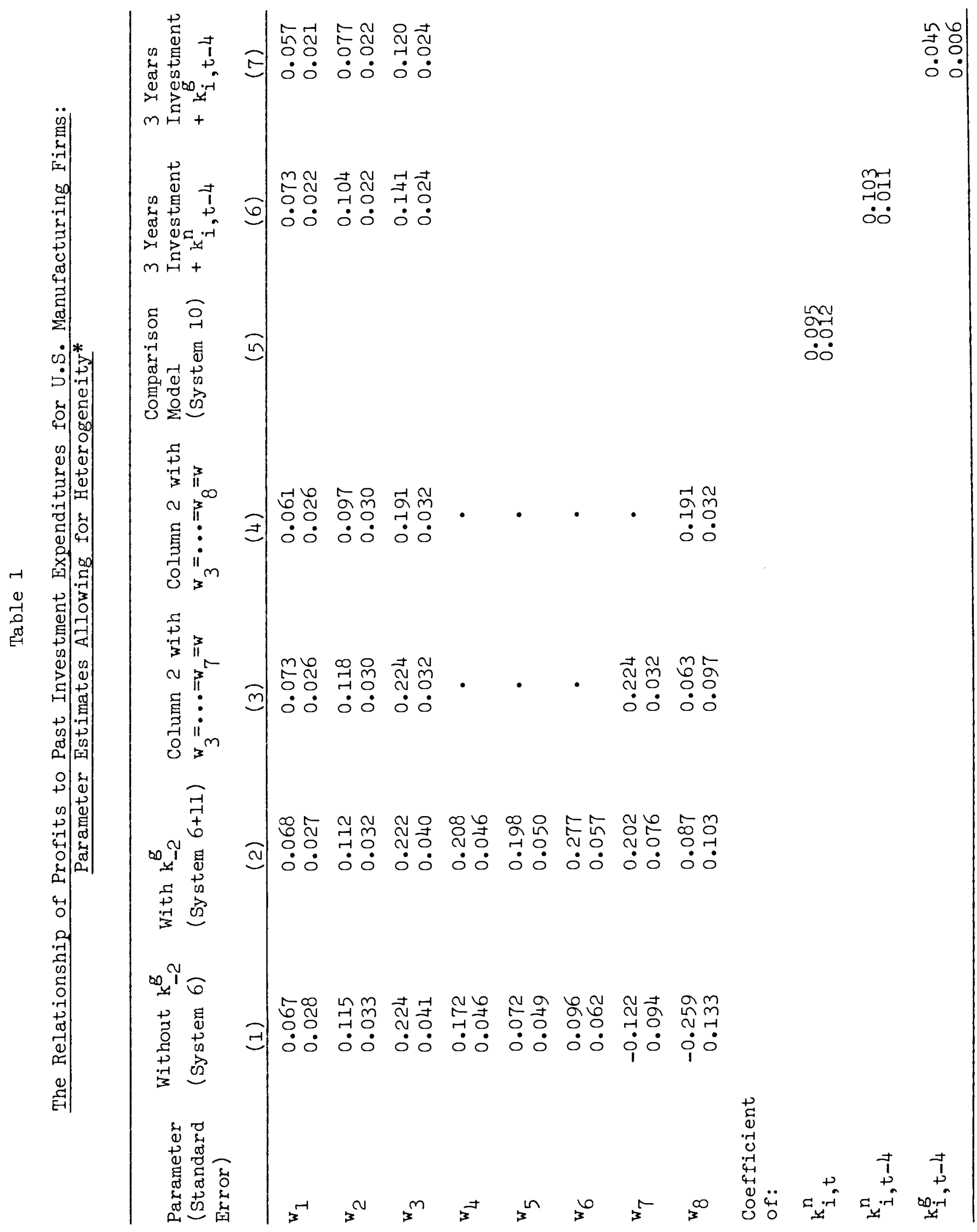




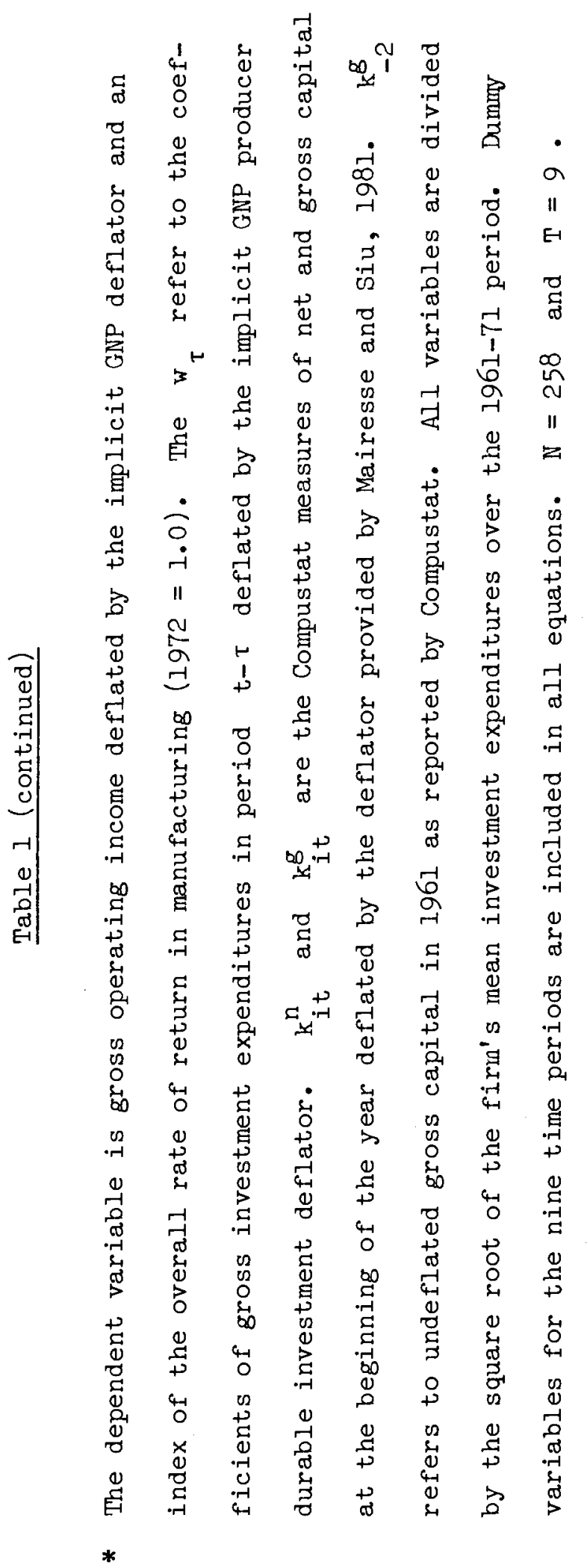


and imposing this constraint resulted in the estimates of column (4). $15 /$ The estimated lag coefficients, then, rise over the first three periods and remain fairly constant over the next four or five. Note that this pattern is distinctly different from the pattern implied by either straight line or declining balance depreciation. Both of these imply coefficients which decline monotonically in $\tau$, and in the second case the decline is greatest in the earlier periods. Column (5) of Table 1 provides the coefficient of $\mathrm{k}_{i t}^{\mathrm{n}}$ obtained from estimating the comparison model (equation 10) while Table 2 provides some measures of goodness of fit. All measures of goodness of fit are based on the trace of the estimated covariance matrices of disturbances from the alternative models, and the traces themselves are presented in row 1 of the table. Rows 2 and 3 normalize, that is divide, row 1 by alternative numbers. In row 3 we divide by $\sum_{t=1}^{T} s_{y t}^{2}$, where $s_{y t}^{2}$ is the sample variance of profits in year $t$, so that one minus the numbers in this row provide an indication of the fraction of the variance in profits over the nine year period accounted for by the model estimated. In row 2 we divide by the trace from a model where the coefficients in the regression of $y_{\sim i}$ on $x_{i}$ are left unconstrained; that is the model which allows for a free II matrix. Comparing the first two columns of the table we see that the trace for the comparison model (which uses $A$ and $k_{i t}^{n}$ as defined by Compustat) is about 75 percent larger that the trace of the model underlying

15. A test of the constraint that $w_{\tau}=\delta_{\tau-1}$ for $\tau \geq 8$ was strongly rejected by the data. 


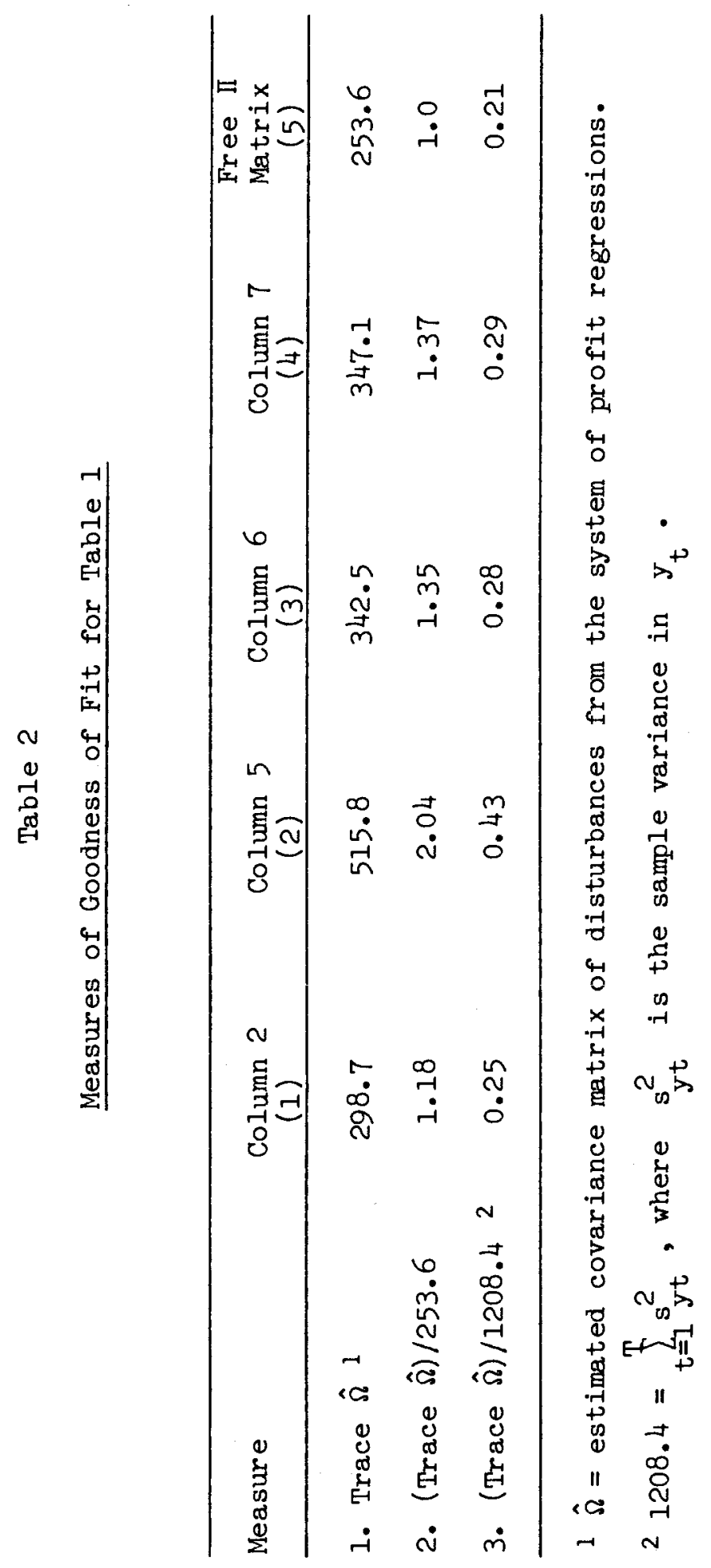


the estimates of colurn (2) in Table 1 (which use Al and A4 of Section II). The latter figure is still, however, 18 percent larger than the trace of a model which allows for a free II matrix (the trace of the comparison model is 104 percent larger than the trace from the model with the free matrix). The model with a free $\pi$ accounts for about 79 percent of the total variance in profits, the model based on $\mathrm{Al}$ and $\mathrm{A} 4$ accounts for about 75 percent of this variance, while the comparison model accounts for about 57 percent of it.

Coming back to the lag structure, recall that the estimates of column (2) suggested that it rose over the first three periods and was fairly flat over the next four or five, with perhaps a slight decline in the last few coefficients estimated. A simple way to approximate such a lag structure might, therefore, be to allow for three free lag coefficients and then substitute either $k_{i t-4}^{g}$ or $k_{i t-4}^{n}$ for $\sum_{\tau=4}^{\infty} w_{\tau} x_{i t-\tau}$ (the choice would depend on the rate of decline in the coefficients which were not estimated). Column (6) (which uses $\mathrm{k}_{i t-4}^{\mathrm{n}}$ ) and column (7) (which uses $\mathrm{k}_{i \mathrm{t}-4}^{\mathrm{g}}$ ) of Table 1 present estimates of these models (the system estimated is constructed in a manner analogous to the construction of the system for the comparison model, see the discussion of system 10). The estimated coefficients in both these columns also rise over the initial three year period. Turning to columns (3) and (4) of Table 2, it is clear that the trace of the estimated coveriance matrices of disturbances for the systems which allow for these three free initial coefficients are much smaller than the trace of the covariance matrix for the comparison model 
(which does not). Their values are only 66 percent of the values of the trace for the comparison model, but they are still over 15 percent larger than the trace of the covariance matrix which uses the estimates of column (2).

So far we have compared the estimates from the model based on a single source of heterogeneity and a set of free lag coefficients to a model which uses capital stock figures from the Cormustat tape, and, in addition, shown that the model's estimates seem to be consistent with the information from auxiliary calculations. Though the model does well in these respects, it does not really provide a completely adequate summary of the data. This becomes evident when we formally test the restrictions on the $I$ matrix embodied in the system consisting of equations (6) and (11). The observed value of the $x_{55}^{2} / 55$ test statistic for the restrictions was 5.64 which indicates rejection of the null hypothesis at traditional levels of significance.16/ Thus though the model underlying the estimates of column (2) seems to provide a better summary of the relationship between past investment and current profits than conventional capital measures, none of these models provide a really adequate account of the interaction between profits and past investment expenditures. This model failure reflects the basic implausibilities in the original maintained hypothesis: (a) that the relationship between investment and profits is essentially the same for different firms and industries and, (b) that

16. Though in judging this test statistic one should keep in mind the rather large size of our sample. 
the time shape of the relationship (the depreciation pattern) is independent of calander time, that the contribution of a particular vintage of investment is independent of the circumstances and factor prices that prevailed at the time it was purchased and from subsequent changes in them. Nevertheless, what is clear is that the usual depreciation schemes which assume that the contribution of past investments declines rapidly and immediately with age are wrong. If anything there may be an "appreciation" in the early years as investments are completed, shaken down, and adjusted to. 


\section{$\underline{\text { References }}$}

* We are grateful to Gary Chamberlain, Lung-fei Lee, Christopher Sims, and to the participants in the Harvard-MIT econometric workshop, and in seminars at the Hebrew University and the NBER, for helpful comments. Gregory Clark and Bronwyn Hall provided capable research assitance and valuable suggestions. This work has been supported by NSF Grants PRA-13740 and SOC79-042479, and by BSF Grant 1881/79. All errors are, of course, solely the authors responsibility.

Anderson, T.W., 1978, Repeated measurements on autoregressive processes, Journal of the American Statistical Association, Vol. 73, $371-378$.

Balestra, P. and M. Nerlove, 1966, Pooling cross section and time series data in the estimation of a dynamic model: The demand for natural gas, Econometrica 34, 585-612.

Box, G.E.P and G.M. Jenkins, 1970, Time series analysis: Forecasting and control, (Holden-Day, San Francisco).

Chamberlain, G., 1978, Analysis of coveriance with qualitative data, Harvard Institute of Economic Research Discussion Paper \#678.

Chamberlain, G., 1982, Multivariate regression models for panel data, Journal of Econometrics 5-46.

Compustat II, 1980, Standard and Poor's Compustat Services, Inc., Englewood, CO.

Dhrymes, P.J., 1971, Distribution lags: Problems of estimation and formulation, (Holden-Day, San Francisco).

Feldstein, M., and L. Summers, 1977, Is the rate of profit falling?, Brookings Papers on Economic Activity, 211-228.

Galbraith, R.F., and J.I. Galbraith, 1974, On the inverses of some patterned matrices arising in the theory of stationary time series, Journal of Applied Probability 11, 63-71.

Geweke, J., and R. Meese, 1979, Estimating regression models of finite but unknown order, Social System Research Institute, The University of Wisconsin, Madison, Discussion Paper \#7925.

Griliches, Z., 1963, Capital stock in investment function: Some problems of concept and measurement, in: C. Christ et. al., Measurement in economics: Essays in memory of Yehuda Grundfeld, (Stanford University Press, Stanford), 115-137.

Griliches, Z., 1967, Distributed lags: A survey, Econometrica 35, 16-49. 
References (continued)

Kiefer, N.M., 1979, Regression systems with fixed effects with a factor structure, May, Unpublished paper.

Kiefer, J. and J. Wolfowitz (1956), Consistency of the maximum likelihood estimator in the presence of infinitely many incidental parameters, Annals of Mathemetical Statistics, Dec., Vol. 27, No. 4, pp. $887-906$.

Klein, L.R., 1958, The estimation of distributed lags, Econometrica, Vol. 26, No. 4, pp. 553-565.

MaCurdy, T.E., 1981, Asymptotic properties of quasi-maximum likelihood estimates and test statistics, Technical Working Paper 14, National Bureau of Economic Research, Cambridge, MA.

MaCurdy, T.E., 1982, The use of time series processes to model the error structure of earnings in a longitudinal data analysis, Journal of Econometrics 18, 83-114.

Maddala, G.S. and A.S. Rao, 1971, Maximum likelihood estimation of Solow's and Jorgenson's distributed lag models, Review of Economics and Statistics 53, pp. 80-88.

Mairesse, J. and A. Siu, 1981, The R\&D and Productivity data master file, unpublished manuscript, National Bureau of Economic Research, Cambridge, MA

Mundlak, Y., 1961, Empirical production function free of management bias, Journal of Farm Economics 43, 44-56.

Mundlak, Y., 1978, On the pooling of time series and cross section data. Econometrica 46, 69-85.

Nerlove, Marc ,1972, Lags in economic behavior, Econometrica 40, $221-251$.

Neyman, Jerzy and E.L. Scott, 1948, Consistent estimates based on partially consistent observations, Econometrica, 1-32.

Pesaran, M.H., 1973, The small sample problem of truncation remainders in the estimation of distributed lag models with autocorrelated errors, International Economic Review 14, 120-131.

Sims, C, 1974, Distributed lags, in: Intriligator and Kendrick (eds.) Frontiers of Quantitative Economics, Vol II, (North-Holland, Amster dam and New York).

Swamy, P.A.V.B., 1970, Efficient inference in a random coefficient regression model, Econometrica 38, 83-114. 
$\underline{\text { References (continued) }}$

Usher, D., (editor), 1980, The measurement of capital, Studies in Income and Wealth, 45, National Bureau of Economic Research (University of Chicago Press, Chicago and London).

White, H., 1980, Using least squares to approximate unknown regression functions, International Economic Review 21, 149-170.

White, H., 1980a, Nonlinear regression on cross-section data, Econometrica 48, 721-746.

White, H., 1980b, A heteroskedasticity-consistent covariance matrix estimator and a direct test for heteroskedasticity, Econometrica $48,817-838$.

White, H., 1982, Instrumental variable regression with independent observations, Econometrica 50, 483-500. 